\title{
Antenatal betamethasone improves postnatal transition in late preterm lambs with persistent pulmonary hypertension of the newborn
}

\author{
Girija G. Konduri', Ivane Bakhutashvili' ${ }^{1}$ Annie Eis ${ }^{1}$ and Adeleye Afolayan ${ }^{1}$
}

BACKGROUND: Persistent pulmonary hypertension of the newborn (PPHN) is associated with increased oxidative stress in pulmonary arteries (PAs). Betamethasone decreases the oxidative stress and improves antioxidant balance in PPHN. We investigated whether antenatal betamethasone improves pulmonary vasodilation and postnatal oxygenation in late preterm lambs with PPHN.

METHODS: PPHN was induced by constriction of fetal ductus arteriosus from 128 to $136 \mathrm{~d}$ gestation. Ewes were given two intramuscular doses of betamethasone or saline at 24 and $12 \mathrm{~h}$ before cesarean-section delivery at $136 \mathrm{~d}$ gestation, simulating late preterm birth. Newborn lambs were mechanically ventilated for $8 \mathrm{~h}$ with monitoring of blood gas and hemodynamic variables. Lungs were harvested postmortem to determine oxidative stress markers and in vitro responses of PAs.

RESULTS: Postnatal arterial partial pressure of oxygen and $\mathrm{pH}$ were higher and the oxygenation index and arterial partial pressure of carbon dioxide were lower in betamethasone-treated lambs. PA pressure was lower and systemic pressure higher in lambs treated with betamethasone. Betamethasone decreased the oxidative stress markers and increased endothelial nitric oxide synthase expression in ventilated PPHN lungs.

CONCLUSION: Antenatal betamethasone decreases oxidative stress and improves postnatal transition in late preterm lambs with PPHN. This study suggests a potential benefit for antenatal betamethasone in late preterm births.

P ersistent pulmonary hypertension of the newborn (PPHN) occurs when the pulmonary vascular resistance fails to decrease at birth (1), resulting in a failure to establish oxygenation by the lung. Infants with PPHN develop hypoxemia and increased risk of death and long-term disabilities (1-3). Late preterm birth is an important cause of respiratory failure and PPHN in newborn infants (4-6). PPHN occurs in association with surfactant deficiency and ventilation/perfusion mismatch in late preterm gestation neonates (6). Although advances in neonatal care decreased the mortality rate of affected infants, survivors of PPHN continue to have increased long-term disability rates $(3,7)$. Current treatment strategies for PPHN target the infants who already have hypoxemia and cardiopulmonary instability inherent to the course of these critically ill neonates. In addition, ventilation with high fractional inspired $\mathrm{O}_{2}$ concentration $\left(\mathrm{FiO}_{2}\right)$, even for a short duration, leads to oxidative stress and sustained vascular dysfunction in the newborn (8). Therefore, improving outcomes in this population may require application of antenatal therapies that facilitate normal adaptation of the lung and decrease lung injury in infants at risk for PPHN.

Previous studies in a fetal lamb model of PPHN induced by prenatal ligation of ductus arteriosus demonstrated that an increase in oxidative stress $(9,10)$ underlies the vascular dysfunction (9-12) in pulmonary arteries (PAs). The vascular dysfunction evolves antenatally and interferes with the transition of pulmonary circulation at birth. Postnatal application of superoxide dismutase (SOD) as a rescue therapy improves pulmonary vasodilation and oxygenation in this model of PPHN $(13,14)$. However, a strategy to correct the vascular dysfunction prenatally in preparation for birth-related transition is not currently available.

Our previous studies demonstrated that the glucocorticoid betamethasone decreases superoxide levels and increases the expression of endothelial nitric oxide synthase (eNOS) and manganese SOD (MnSOD) and the bioavailability of nitric oxide (NO) in the PA endothelial cells in PPHN lambs (15). Antenatal administration of betamethasone improves the in vitro relaxation response of PAs isolated from unventilated lungs of both normal and PPHN fetal lambs $(15,16)$. Corticosteroids decrease oxidative stress in the presence of lung inflammation in asthma (17). Previous studies in fetal rats and lambs demonstrated that prenatal steroids induce an increase in antioxidant enzyme activity and expression (18-20). We proposed the hypothesis that antenatal betamethasone administration would improve postnatal pulmonary vasodilation and oxygenation in PPHN by decreasing oxidative stress in the lung. We tested the effects of antenatal betamethasone following a clinically used dosing regimen that was modified to minimize the incidence of preterm labor in fetal lambs. We conducted the studies in intact lambs delivered at late preterm gestation after the prenatal induction of PPHN. 

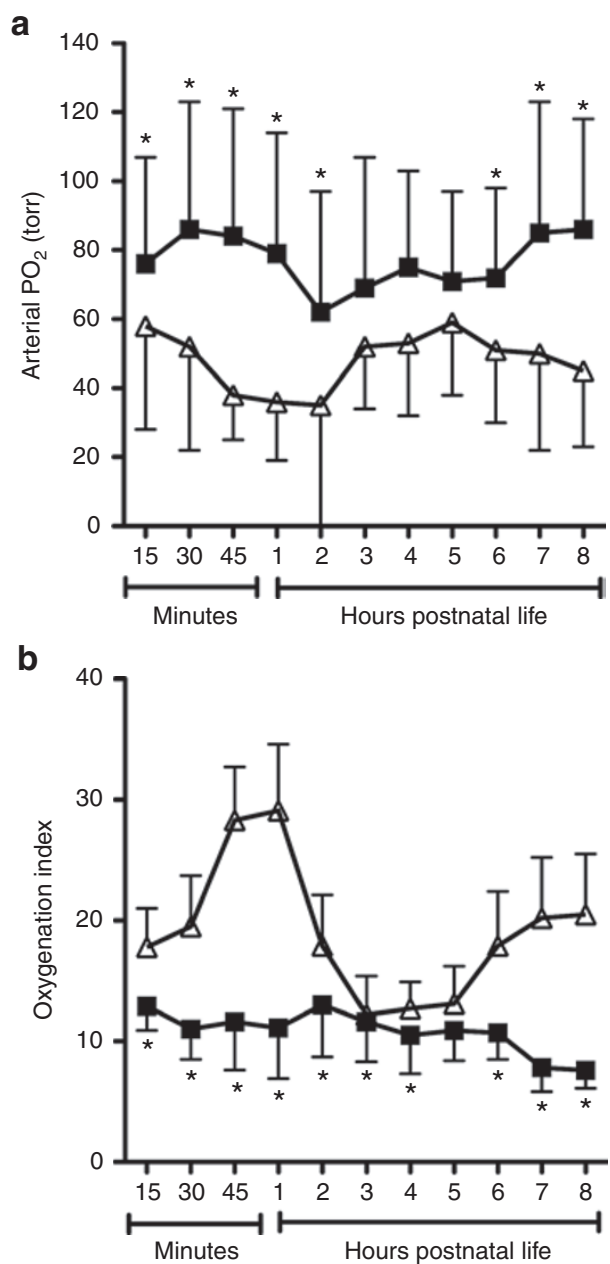

Figure 1. Effect of betamethasone on (a) postnatal $\mathrm{PaO}_{2}$ and (b) oxygenation index. Data are mean \pm SD for six animals in each group. Filled squares indicate data for betamethasone-treated PPHN group and open triangles for saline-treated PPHN group (control). ${ }^{*} P<0.05$, as compared with control. Betamethasone-treated lambs had higher $\mathrm{PaO}_{2}$ and lower oxygenation index during postnatal ventilation. PPHN, persistent pulmonary hypertension of the newborn.

\section{RESULTS}

A total of 20 fetal lambs, 10 in each group, had PPHN induced; six control and six betamethasone-treated lambs each completed the $8 \mathrm{~h}$ of ventilation. Three animals in the control group and one in betamethasone group died before the completion of $8 \mathrm{~h}$ of ventilation. Three ewes in the betamethasone group and one control ewe had preterm labor before cesarean-section (C-section) delivery of the fetus. In addition, three unventilated fetal lambs that had exposure to either saline or betamethasone and three normal term lambs that were ventilated were included for immunoblotting or vascular ring studies.

Betamethasone-treated lambs had significantly better oxygenation during the first $2 \mathrm{~h}$, with a twofold difference by the end of $1 \mathrm{~h}$ (Figure 1a). The control animals had some increase in the $\mathrm{PO}_{2}$ during hours $2-5$, and the difference between the two groups was not significant during that time. However, betamethasone-treated lambs had higher $\mathrm{PaO}_{2}$ at hours 6-8 as compared with controls, with $40-90 \%$ differences in $\mathrm{PaO}_{2}$. The oxygenation index was also significantly lower in the betamethasone-treated lambs, indicating lower severity of respiratory failure (Figure $\mathbf{1 b}$ ).

Betamethasone-treated lambs had lower $\mathrm{PaCO}_{2}$ at hours $0-2$, 7 , and 8 , indicating that lung compliance probably improved faster in this group as compared with the controls (Figure 2a). Given that the lambs were delivered 7-8 d before term gestation, the improvement in compliance may be due to the beneficial effect of betamethasone on lung fluid clearance and surfactant release (21). The ventilatory index was consistently lower in betamethasone-treated lambs (Figure $\mathbf{2 b}$ ), as the ventilator pressure and rate were weaned for lower $\mathrm{PaCO}_{2}$. The arterial $\mathrm{pH}$ was higher in betamethasone-treated lambs at hours $0-2$ and 6-8 as compared with that of controls (Figure 2c), consistent with lower $\mathrm{PaCO}_{2}$.

The PA pressure was $15 \%$ lower in the betamethasone-treated group during the first hour and hours 3-8 of postnatal ventilation (Figure 3a). The aortic pressure was higher and more stable in the betamethasone-treated lambs, whereas it declined
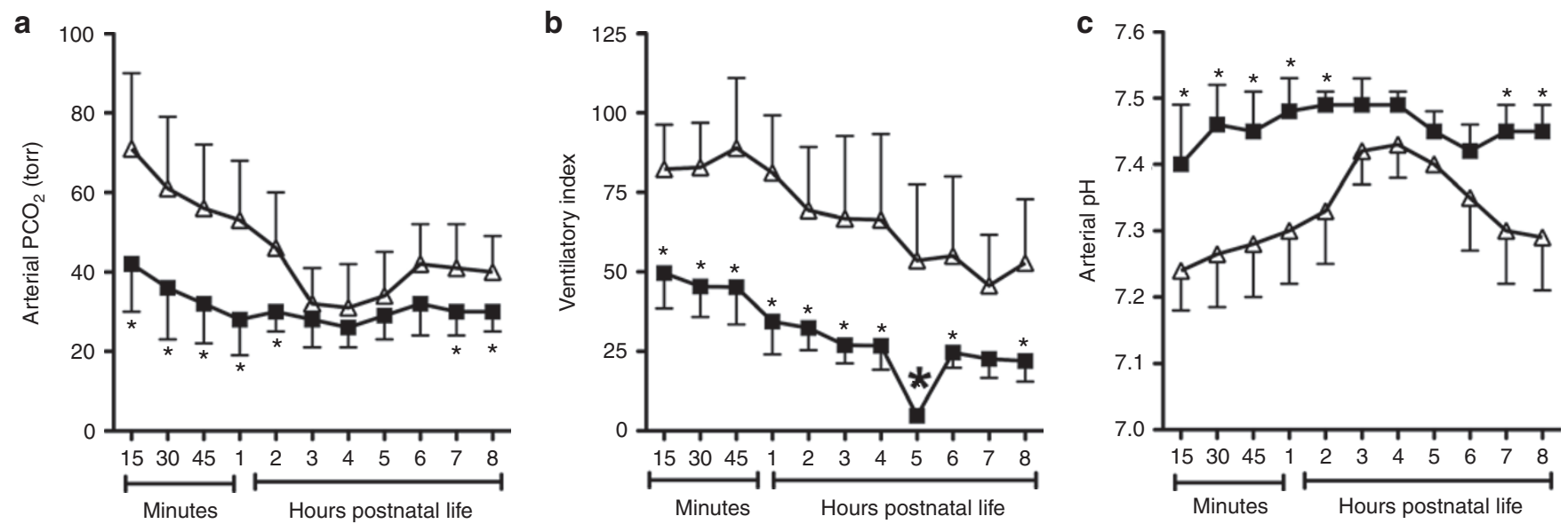

Figure 2. Effect of betamethasone on (a) postnatal $\mathrm{PaCO}_{2}$, (b) ventilatory index, and (c) arterial blood pH. Data are mean $\pm \mathrm{SD}$ for six animals in each group. Filled squares indicate data for betamethasone-treated PPHN group and open triangles for saline-treated PPHN control. ${ }^{*} P<0.05$, as compared with control. Betamethasone-treated lambs had lower $\mathrm{PaCO}_{2}$ and ventilatory index and higher $\mathrm{pH}$ as compared with controls. PPHN, persistent pulmonary hypertension of the newborn. 

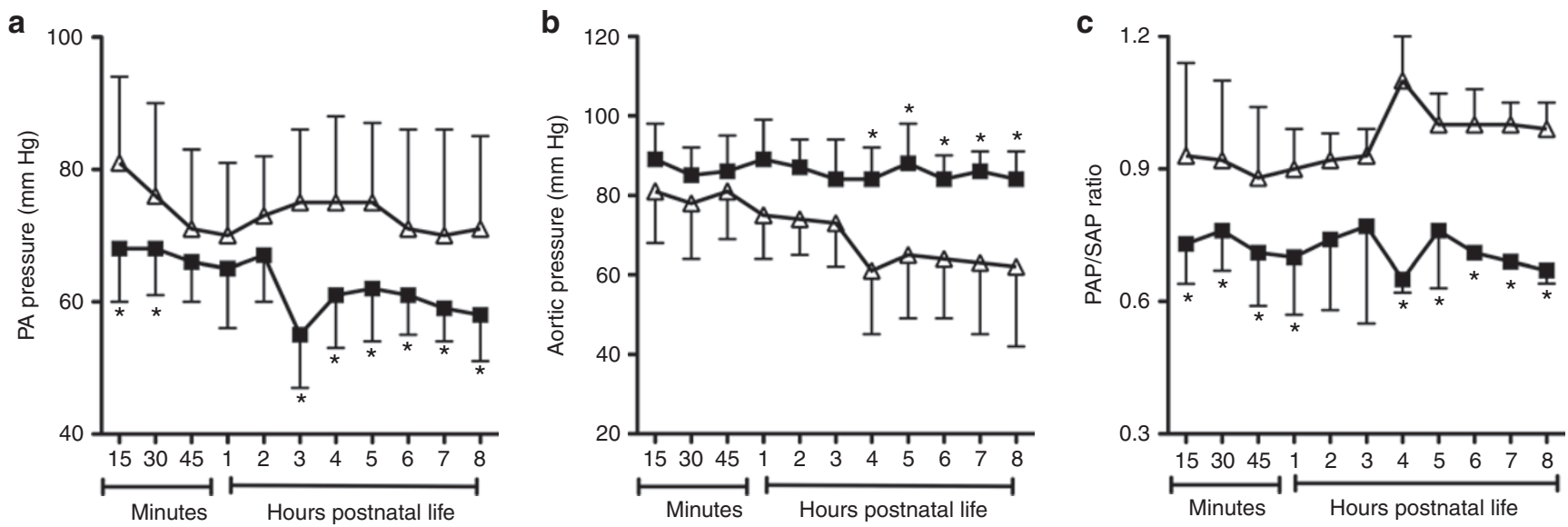

Figure 3. Effect of betamethasone on (a) postnatal pulmonary artery (PA) pressure, (b) aortic pressure, and (c) ratio of PA pressure (PAP) to aortic pressure (SAP) during ventilation. Data are mean \pm SD for six animals in each group. Filled squares indicate data for betamethasone-treated PPHN group and open triangles for saline-treated PPHN control group. ${ }^{*} P<0.05$, as compared with controls. Betamethasone-treated lambs had lower PA pressure, higher aortic pressure, and lower PAP/SAP ratio as compared with controls. PPHN, persistent pulmonary hypertension of the newborn.
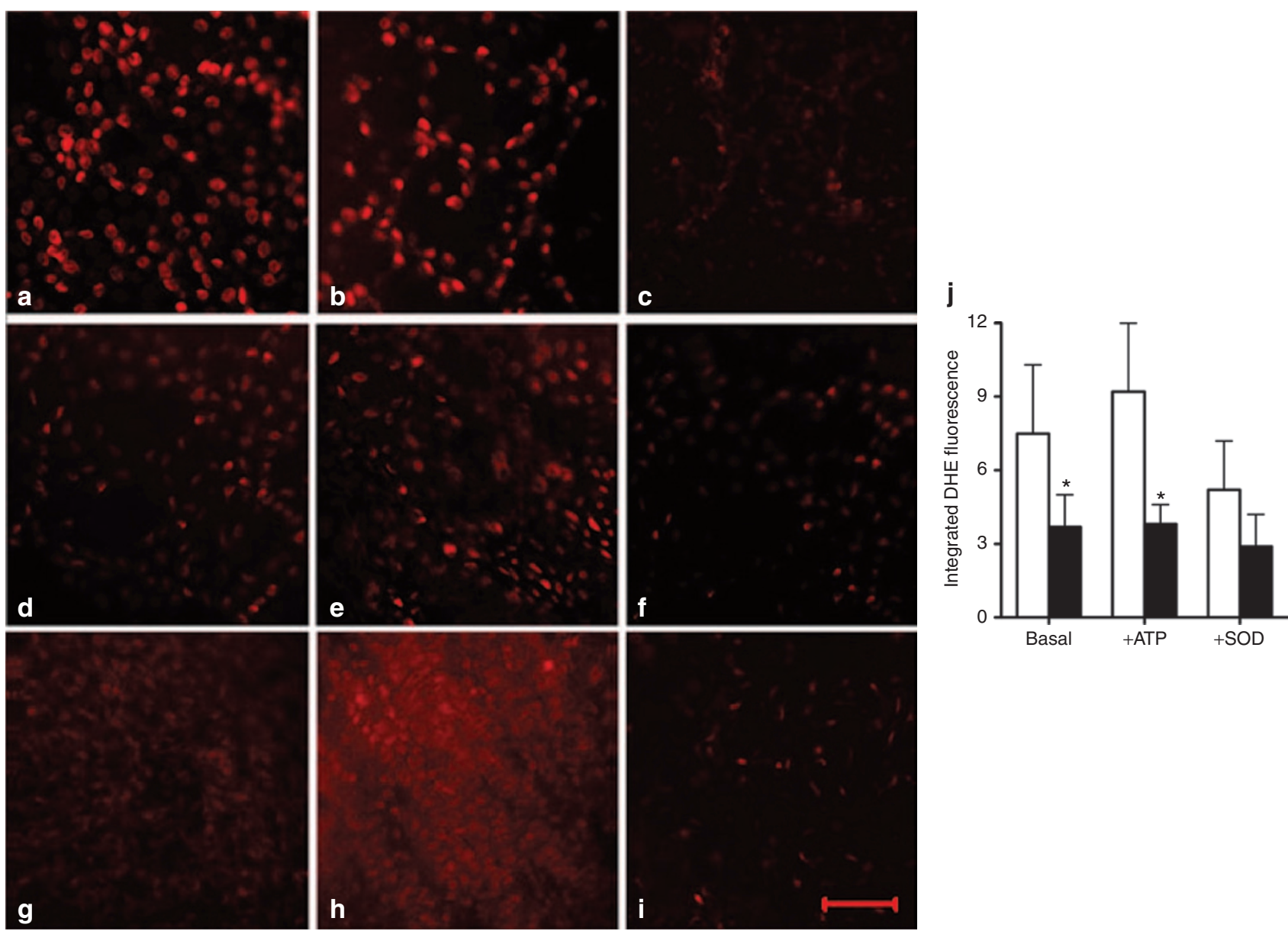

Figure 4. Dihydroethidine (DHE) fluorescence of pulmonary artery endothelium in ventilated PPHN lambs (a-c) without or (d-f) with prenatal betamethasone and $(\mathbf{g}-\mathbf{i})$ a normal fetal lamb control. $(\mathbf{a}, \mathbf{d}, \mathbf{g})$ Representative fluorescence is shown for basal level, (b, e) after ATP stimulation, (h) after exposure to superoxide from xanthine + xanthine oxidase as a positive control, and $(\mathbf{c}, \mathbf{f}, \mathbf{i})$ with superoxide scavenger, polyethylene glycol (PEG)-SOD, as a negative control. Scale bar in (i) represents $50 \mu \mathrm{m}$, and images are at $\times 200$ original magnification. (j) Summarized data are shown for six animals each for no betamethasone (open bars) and betamethasone-treated pulmonary arteries (filled bars) as mean \pm SD. ${ }^{*} P<0.05$, as compared with controls. Betamethasone-treated lambs had lower DHE fluorescence at basal and stimulated conditions, and the fluorescence was quenched by PEG-SOD, indicating contribution of superoxide to DHE fluorescence. ATP, adenosine triphosphate; PPHN, persistent pulmonary hypertension of the newborn. 


\section{Articles | Konduriet al.}
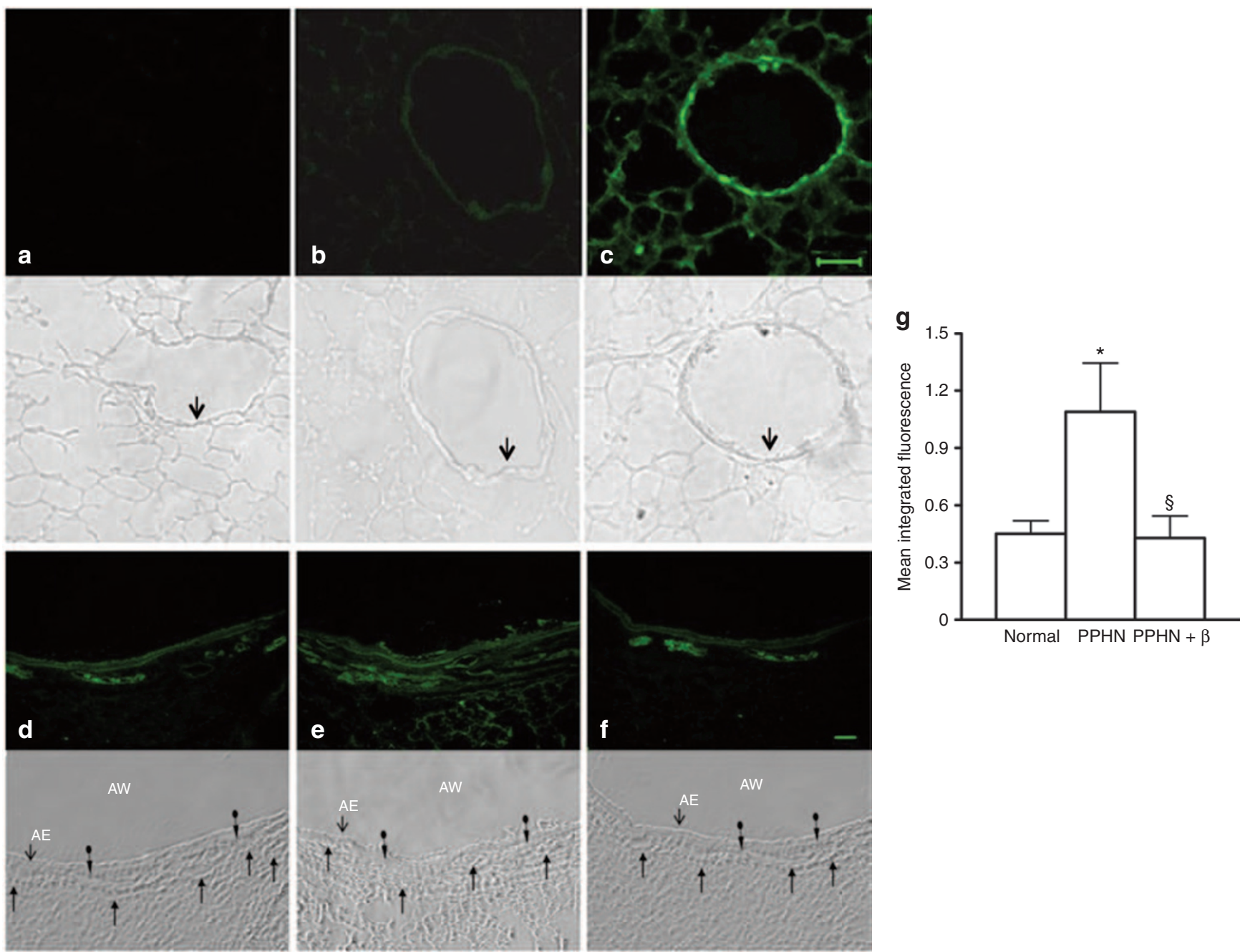

Figure 5. Nitrotyrosine labeling of frozen lung section from (a-c) unventilated and (d-f) ventilated lambs with the corresponding phase-contrast microscopy images shown directly below. For normal lung sections, (a) negative control, antibody preabsorbed with 3-nitro-L-tyrosine before applying to lung section, (b) basal nitrotyrosine immunolabeling, and (c) positive control, lung section pretreated with $5 \mu \mathrm{mol} / \mathrm{l}$ peroxynitrite before nitrotyrosine immunolabeling. Scale bar in (c) is $50 \mu \mathrm{m}$, and original magnification is $\times 200$. Arrows in phase-contrast images below point to airway epithelium. For ventilated lungs, (d) ventilated normal lung, (e) ventilated PPHN lung, and (f) PPHN lamb treated with betamethasone before ventilation. Original magnification is $\times 100$ for panels $(\mathbf{d}-\mathbf{f})$. The plain arrows indicate capillaries and circle arrows indicate airway smooth muscle. Summary data in (g) show increase in nitrotyrosine in PPHN lambs as compared with ventilated normal lambs (normal) and decrease with betamethasone (PPHN $+\beta$ ). ${ }^{*} P<0.05$, as compared with normal; ${ }^{5} P<0.05$, as compared with PPHN lambs without betamethasone. Betamethasone-treated PPHN lambs had lower nitrotyrosine levels in the lung sections. $A E$, airway epithelium; $A W$, airway; $P P H N$, persistent pulmonary hypertension of the newborn.

in the control lambs from hour 4 to hour 8 (Figure 3b). The PA pressure /aortic pressure ratio was also lower in betamethasone-treated lambs during the first hour and hours $4-8$ of postnatal ventilation (Figure $3 \mathrm{c}$ ).

PAs from ventilated PPHN lambs had high dihydroethidine (DHE) fluorescence at the basal level (Figure 4a) and after stimulation with adenosine triphosphate (ATP) (Figure 4b); the fluorescence was quenched by polyethylene glycol-SOD (Figure 4c). Betamethasone-treated animals had lower DHE fluorescence at the basal level (Figure 4d,j) and after the stimulation of PA segments with ATP, which increased the intracellular calcium levels (Figure 4e,j). Superoxide from xanthine + xanthine oxidase markedly increased the fluorescence in normal PAs (Figure $4 \mathrm{~h}$ ) as expected, and the fluorescence was quenched by polyethylene glycol-SOD (Figure 4i), indicating that changes in superoxide $\left(\mathrm{O}_{2}^{-}\right)$levels were responsible for the differences in DHE fluorescence in PPHN and after betamethasone treatment.

The 3-nitrotyrosine (3-NT) levels were low in unventilated lamb lungs (Figure 5b); exposure of lung section to peroxynitrite markedly increased the signal for $3-\mathrm{NT}$, as expected (Figure 5c). 3-NT levels were higher in the ventilated PPHN lambs (Figure 5e) as compared with either normal ventilated lamb lungs (Figure 5d) or betamethasone-treated ventilated lambs with PPHN (Figure 5f). The signal was higher in both airway and PAs in PPHN lambs (Figure 5e). Betamethasone decreased the signal for 3-NT in both airway and PAs in ventilated PPHN lambs (Figure 5f,g).

The levels of MnSOD, copper zinc SOD, and extracellular SOD in the unventilated normal lungs were increased by $50 \%$ with prenatal exposure to betamethasone (Figure $6 \mathrm{a}, \mathrm{b}$ ). 


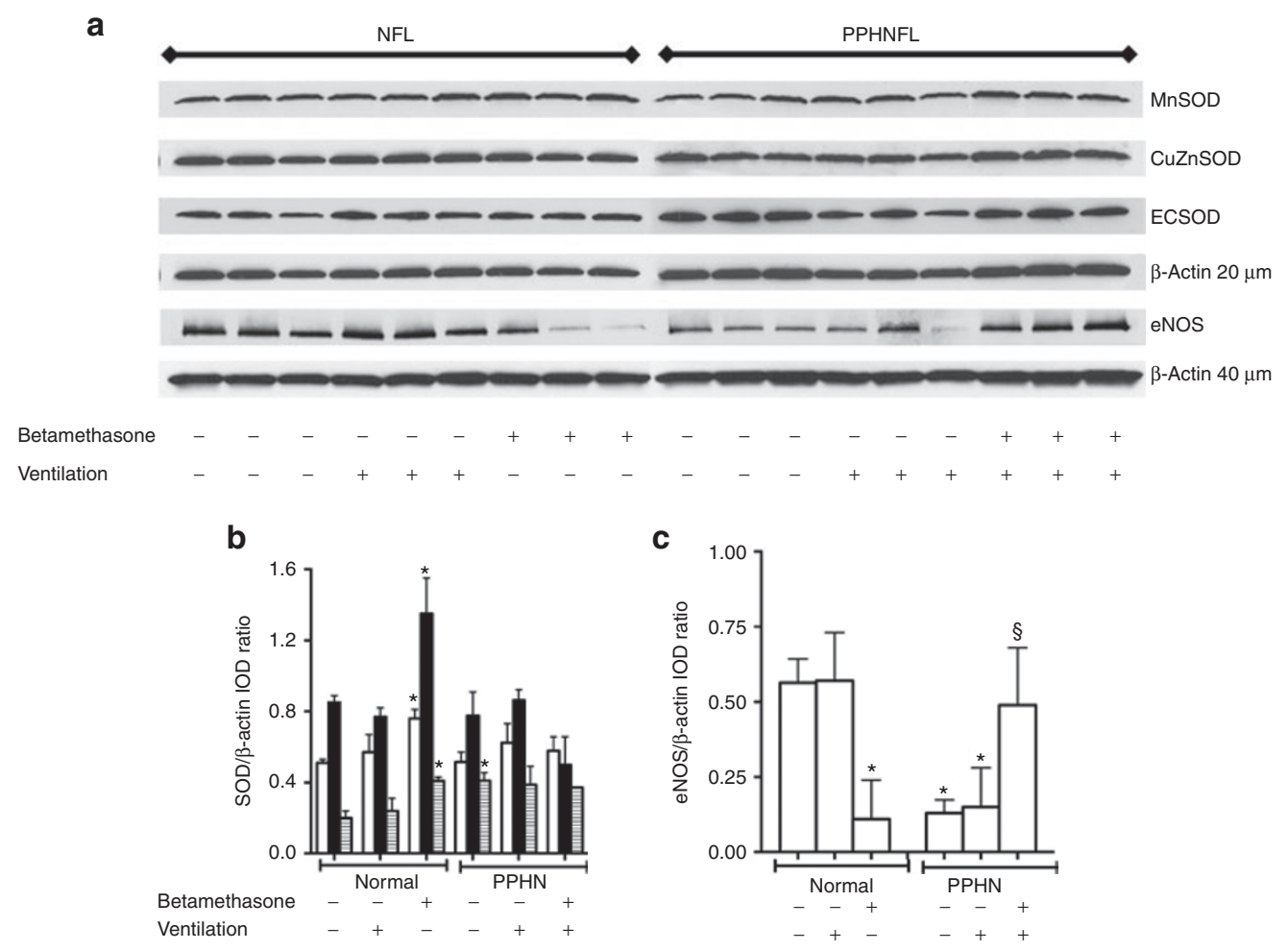

Figure 6. Effect of betamethasone on the expression of SOD isoforms and eNOS in the lungs of fetal and neonatal lambs. (a) Representative immunoblots showing protein levels of MnSOD, CUZnSOD, ECSOD, and eNOS with $\beta$-actin as internal control. Blots are shown for samples from unventilated and ventilated normal fetal lambs (NFL) and PPHN lambs (PPHNFL) with or without betamethasone as indicated on the figure labels. Twenty micrograms of protein was loaded for SOD blots and corresponding $\beta$-actin, and $40 \mu \mathrm{g}$ was loaded for eNOS and corresponding $\beta$-actin. Summarized data for integrated optical densities (IODs) of (b) SOD/ $\beta$-actin ratio and (c) eNOS/ $\beta$-actin ratio are shown as mean \pm SD for three lambs each. In (b), open bars are for MnSOD, filled bars for CuZnSOD, and hatched bars for ECSOD. ${ }^{*} P<0.05$, as compared with normal unventilated lambs without betamethasone; ${ }^{\S} P<0.05$, as compared with PPHN ventilated lambs without betamethasone. CuZnSOD, copper zinc superoxide dismutase; ECSOD, extracellular superoxide dismutase; MnSOD, manganese superoxide dismutase; PPHN, persistent pulmonary hypertension of the newborn.

The protein levels of MnSOD and copper zinc SOD did not change further with PPHN or with ventilation in either saline- or betamethasone-exposed lambs, whereas extracellular SOD expression was higher in PPHN unventilated lungs. Expression of eNOS was decreased in unventilated betamethasone-exposed normal lungs (Figure 6a,c) and was also fourfold lower in PPHN ventilated and unventilated lungs, as previously reported $(9,11)$. Prenatal exposure to betamethasone increased the eNOS expression by threefold in the ventilated PPHN lungs (Figure 6a,c).

PA rings isolated from betamethasone-treated lambs showed increased contractile responses to both norepinephrine and potassium chloride $(\mathrm{KCl})$ (Figure 7a,b) as compared with saline-treated PPHN lambs. Control rings from normal term ventilated lambs showed higher constrictor response at $118 \mathrm{mmol} / \mathrm{l} \mathrm{KCl}$ as compared with other groups, which may be related to their greater maturity and smooth muscle mass (Figure 7a). The relaxation responses to NOS agonist, ATP, and NO donor $S$-nitroso- $N$-acetyl penicillamine were similar between the two PPHN groups (Figure 7c,d).

\section{DISCUSSION}

We provide evidence that antenatal betamethasone improves oxygenation and facilitates pulmonary vasodilation in a model of PPHN in newborn lambs delivered at late preterm gestation. The effects of betamethasone were sustained for the 8-h study period and were accompanied by decreased markers of oxidative stress in the airway and PAs. Betamethasone also induced an increase in the levels of eNOS in the ventilated PPHN lungs. These data suggest that antenatal betamethasone can benefit postnatal transition in the presence of PPHN at late preterm gestation. We used both $\mathrm{O}_{2}^{-}$and nitrotyrosine as markers of oxidative stress because previous studies demonstrated an upregulation of nicotinamide adenine dinucleotide phosphate oxidase, a source of $\mathrm{O}_{2}^{-}$, and uncoupling of eNOS $(10,12)$. Uncoupled eNOS generates both $\mathrm{NO}$ and $\mathrm{O}_{2}^{-}$, which combine to form peroxynitrite, a free radical that nitrates tyrosine residues. We observed an increase in nitrotyrosine with PPHN + ventilation and a reduction in this marker with betamethasone.

Betamethasone has several physiological effects on the preterm lung including a decrease in lung fluid, an increase in antioxidant enzyme activity and expression (18-20), and surfactant release (21). Our previous studies demonstrated that betamethasone increases the expression of both eNOS and MnSOD in fetal PA endothelial cells when added to culture media for $48 \mathrm{~h}$ (15). These changes were accompanied by a decrease in the superoxide and increase in NO levels (15). However, the potential benefit of betamethasone in facilitating 


\section{Articles | Kondurietal.}

a

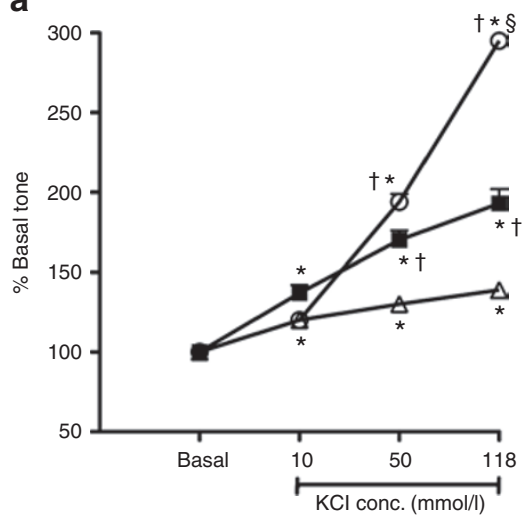

b

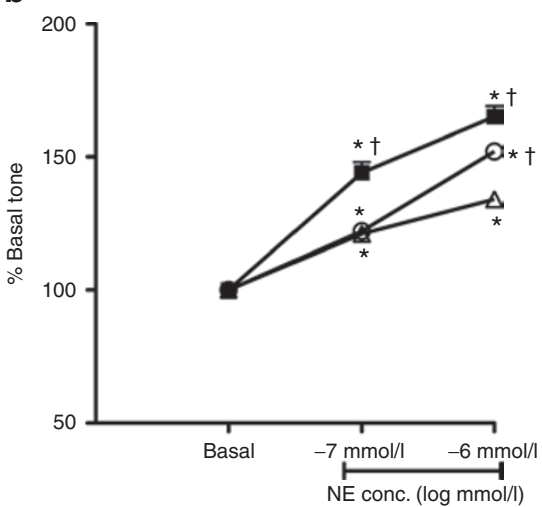

C

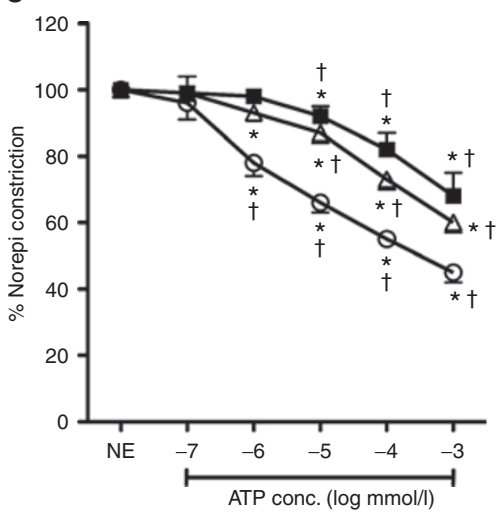

d

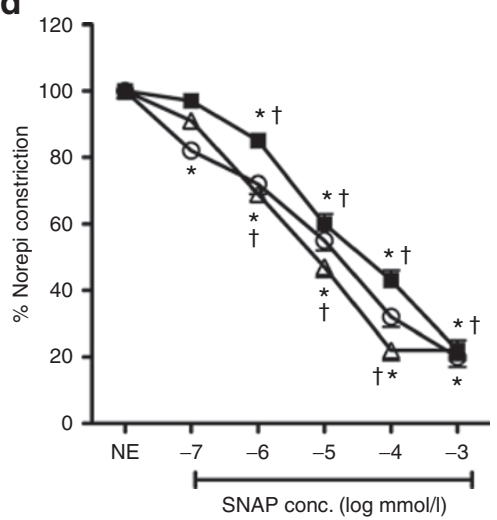

Figure 7. Effect of betamethasone on the response of isolated pulmonary arteries. (a, $\mathbf{b})$ Contraction and $(\mathbf{c}, \mathbf{d})$ relaxation responses of pulmonary artery rings from ventilated normal (open circle), PPHN (open triangle), and PPHN + betamethasone (filled square) lamb lungs. Data are mean \pm SEM for 12 rings for normal lambs and 24 rings for PPHN groups with or without betamethasone. For potassium chloride (KCl) and norepinephrine (NE) contraction responses, basal tone of each ring was normalized to $100 \%$. For relaxation responses $(\mathbf{c}, \mathbf{d})$ ), tone achieved after norepinephrine (Norepi) constriction was normalized to $100 \%$ for each ring. For panels $(\mathbf{a}, \mathbf{b}),{ }^{*} P<0.05$ as compared with basal tone; ${ }^{\dagger} P<0.05$, as compared with PPHN group without betamethasone; ${ }^{5} P<0.05$, as compared with betamethasone-treated group. Betamethasone enhanced the contractile response of rings to $\mathrm{KCl}$ and norepinephrine, whereas rings from normal lambs achieved greater tone at a maximal dose of $\mathrm{KCl}$ as compared with both the PPHN groups. For panels $(\mathbf{c}, \mathbf{d}),{ }^{*} P<0.05$, as compared with $\mathrm{NE}^{+}{ }^{+} P<0.05$, as compared with preceding dose of ATP or $S$-nitroso- $N$-acetylpenicillamine (SNAP). ATP and SNAP induced comparable relaxation response in all three groups. ATP, adenosine triphosphate; conc., concentration; PPHN, persistent pulmonary hypertension of the newborn.

the postnatal transition in intact fetal lambs was not investigated in our previous in vitro studies.

Previous studies also reported that antenatal glucocorticoids facilitate birth-related transition (16,22-24). Antenatal steroids increased pulmonary vasodilation and expression of eNOS in lamb lungs at birth (22-24). We observed an increase in the expression of three SOD isoforms in the unventilated normal fetal lungs with betamethasone in the current study. However, we did not observe a change in SOD expression with betamethasone in ventilated PPHN lungs. It is likely that ventilation with the high $\mathrm{FiO}_{2}$ necessary to sustain $\mathrm{PaO}_{2}$ in $\mathrm{PPHN}$ lungs disrupted this effect of betamethasone. Another possible explanation for the difference between our in vitro data and the current study is that we gave betamethasone $24 \mathrm{~h}$ before delivery, resulting in a shorter exposure time. We previously observed a decrease in MnSOD expression in PA endothelial cells from PPHN lambs (15). Farrow et al. (25) reported an increase in MnSOD expression and activity in vascular smooth muscle cells isolated from PPHN lambs. Our current studies demonstrate no change in the total lung expression of MnSOD in PPHN, probably as a result of these reciprocal changes in different cells. These data also suggest that changes in SOD expression may be cell specific in PPHN. In addition, we did not measure the total or isoform-specific SOD activity in our studies, which may be altered by PPHN or betamethasone. Betamethasone decreased basal eNOS expression in unventilated normal lungs but increased the eNOS expression in ventilated PPHN lamb lungs, consistent with our in vitro data from PA endothelial cells (15). However, the increase in eNOS expression in the lung was not accompanied by improved relaxation response of PAs to the NOS agonist, ATP. These data suggest that function of eNOS is still impaired, probably from prolonged ventilation of $\mathrm{PPHN}$ lambs with high $\mathrm{FiO}_{2}$.

Our model of fetal ductal constriction is known to cause PPHN in lambs similar to findings observed in neonates with PPHN $(26,27)$. We also delivered the lambs in our study by C-section 1 week before term gestation. The lambs in our study were not given exogenous surfactant. This model therefore reproduces the respiratory failure from both lung immaturity and PPHN. Currently, late preterm births are an important cause of respiratory failure, leading to PPHN in neonates. Walsh-Sukys et al. (2) observed that $32 \%$ of babies diagnosed with PPHN 
were delivered by $\mathrm{C}$-section before the onset of labor in a large cohort from the National Institute of Child Health and Human Development Neonatal Research Network. C-section delivery before the onset of labor was noted to increase the risk of PPHN significantly in other studies $(4,5)$. Late preterm gestation neonates with respiratory failure have increased morbidity and mortality as compared with term infants with a comparable degree of respiratory failure (6). Our study suggests that antenatal betamethasone decreases the oxidative stress and improves oxygenation at comparable gestation.

The improvement in $\mathrm{PaO}_{2}$ in our study was not significant at hours 3-6. This is due to some increase in $\mathrm{PO}_{2}$ in control lambs and weaning of $\mathrm{FiO}_{2}$ in betamethasone-treated lambs. The oxygenation index remained consistently low and $\mathrm{PO}_{2}$ remained consistently above 50 in betamethasone-treated lambs. The data also showed variability inherent to the ventilation studies and small sample size typical of these complex studies (28). The PA pressure decreased in betamethasone-treated animals, matching the improvement in oxygenation. In addition, the $\mathrm{PaCO}_{2}$ was lower and $\mathrm{pH}$ higher in betamethasone-treated lambs. These data indicate that improvement in lung mechanics may also contribute to improved oxygenation in this model. Because an increase in $\mathrm{pH}$ and decrease in $\mathrm{PCO}_{2}$ can reduce PA pressure, the effect of betamethasone on these variables may have contributed to pulmonary vasodilation independent of its effects on the oxidative stress. The systemic pressure was consistently higher in betamethasone-treated lambs. These results suggest that an increase in cardiac output may also contribute to the improved oxygenation in betamethasone-treated lambs. The in vitro relaxation response of PAs was not improved by betamethasone. The mechanism of improved oxygenation in betamethasone-treated lambs remains unclear from the in vitro studies.

A recent study reported that administration of postnatal hydrocortisone to lambs with PPHN induced by prenatal ductal constriction improves oxygenation and decreases oxidative stress in the lung during mechanical ventilation (28). PA pressure changes were not reported, and it is unclear whether improved oxygenation resulted from pulmonary vasodilation or improved lung mechanics. Our data are overall consistent with the reported effects of postnatal hydrocortisone, and both approaches offer complementary strategies to decrease oxidative stress and improve oxygenation in PPHN.

The limitation of our study is that the period of postnatal ventilation was limited to $8 \mathrm{~h}$. We chose this time point because of high mortality in the control lambs with PPHN after $8 \mathrm{~h}$ of ventilation. We also observed that betamethasone increased the contractile response of the PAs to norepinephrine and $\mathrm{KCl}$. This is a well-known effect of steroids in systemic vessels and involves calcium sensitization of smooth muscle cells. Whether a similar mechanism accounts for the observed PA responses is unclear. Although increased contractility may appear to counter pulmonary vasodilation at birth, it may enhance oxygenation by preserving vasoconstriction in the unventilated segments of the lung and promoting ventilation/perfusion matching.

In conclusion, we observed that antenatal betamethasone improves oxygenation and pulmonary vasodilation during postnatal life in newborn lambs with PPHN delivered at late preterm gestation. Our study supports the potential clinical use of antenatal betamethasone for indicated deliveries at late preterm gestation.

\section{METHODS}

The Institutional Animal Care and Use Committee of the Medical College of Wisconsin approved the use of animals for studies described in this article.

\section{Creation of PPHN Model}

Pregnant ewes of Dorset Cross or Speckled Face breed were obtained from Purdue University (West Lafayette, IN) at $118 \pm 4 \mathrm{~d}$ of gestation; average full-term gestation for sheep is $145 \mathrm{~d}$. Ewes underwent midline laparotomy and hysterotomy under general anesthesia at $128 \pm 2 \mathrm{~d}$ of gestation. Fetal chest was exteriorized, and a left lateral thoracotomy was performed for constriction of ductus arteriosus, as previously reported $(11,12,29)$. A catheter was placed in the PA by direct puncture, and the tip was directed into the left PA during the same procedure. The ductal constriction was maintained for $8 \mathrm{~d}(128 \pm 2$ to $136 \pm 2 \mathrm{~d})$. Catheters were placed in the superior vena cava and aortic arch through left jugular vein and common carotid artery at the time of C-section delivery.

Ewes were randomly assigned to either a treatment group that received betamethasone or a control group that received an equal volume of saline by i.m. injection. Betamethasone (Celestone; MerckSchering Plough, Whitehouse Station, NJ) was given in two doses of $12 \mathrm{mg}$ each, 24 and $12 \mathrm{~h}$ before C-section delivery of the fetal lamb at $136+2$ days of gestation. Administration of betamethasone more than $24 \mathrm{~h}$ before planned delivery has led to induction of preterm labor, as previously reported (30). Fetal lambs were delivered by C-section under general anesthesia with 1-3\% isoflurane inhalation.

Three fetal lambs without PPHN and without betamethasone exposure and three twin fetal lambs without PPHN and exposed to antenatal betamethasone were also included as additional controls for in vitro studies. Three term fetal lambs were also ventilated for $8 \mathrm{~h}$ as additional controls for in vitro studies.

\section{Ventilation of Fetal Lambs}

The fetal trachea was intubated with a 4.0 -cuffed endotracheal tube, and the cuff was inflated to prevent air leak. Fetal lung liquid was suctioned to improve ventilation. Ventilation was commenced after the umbilical cord was tied and cut, and the newborn lamb was placed on a warmer. Lambs were sedated with intermittent doses of pentobarbi$\mathrm{tal}(5 \mathrm{mg} / \mathrm{kg}$ intravenously) and buprenorphine $(0.005 \mathrm{mg} / \mathrm{kg}$ i.m.) and paralyzed with vecuronium $(0.1 \mathrm{mg} / \mathrm{kg}$ i.v. and repeated as indicated). Lambs were given $10 \%$ dextrose i.v. at $4 \mathrm{ml} / \mathrm{kg} / \mathrm{h}$ to keep blood glucose concentration in the normal range. Surfactant was not used in order to simulate the typical respiratory transition in late preterm newborn at birth and to avoid confounding the effects of betamethasone on respiratory failure. Ventilation was commenced with an Infant Star timecycled pressure-limited ventilator (Infrasonics, Sorrento Valley, CA) with peak inspiratory pressure of $25-35 \mathrm{~cm} \mathrm{H}_{2} \mathrm{O}$, positive end expiratory pressure of $5-6 \mathrm{~cm} \mathrm{H}_{2} \mathrm{O}$, frequency of $30-40$ breaths/min, inspiratory time of $0.4-0.5 \mathrm{~s}$, and $\mathrm{FiO}_{2}$ of $50 \%$, as reported previously (14). The ventilator settings were selected to keep arterial $\mathrm{PaCO}_{2}$ in a target range of $40-55 \mathrm{~mm} \mathrm{Hg}, \mathrm{PaO}_{2}$ in a range of $50-70 \mathrm{~mm} \mathrm{Hg}$, and $\mathrm{pH}>$ 7.20. The ventilator settings were not changed after the target blood gas tensions were reached. Arterial blood gas tensions and blood $\mathrm{pH}$ were measured at $15,30,45$, and $60 \mathrm{~min}$ and every hour for the $8 \mathrm{~h}$ of ventilation. Systemic and PA pressures and heart rate were continuously monitored. Oxygenation index was calculated using the formula: mean airway pressure $\times \mathrm{FiO}_{2} \times 100 \div \mathrm{PaO}_{2}$, and ventilatory index was calculated using the formula: respiratory rate $\times$ (peak inspiratory pressure - positive end expiratory pressure $) \times \mathrm{PaCO}_{2} \div 1,000$.

\section{PA Ring Studies}

After the ventilation studies were completed, lambs were killed with an overdose of pentobarbital. Lungs were harvested, and 
third-fifth-generation intra-PAs with an internal diameter of 300-500 $\mu \mathrm{mol} / \mathrm{l}$ were dissected and isolated from the lung. The arteries were cut into rings $1 \mathrm{~mm}$ in length, suspended with stainless steel hooks in waterjacketed chambers, and studied using standard tissue bath techniques (12). They were allowed to equilibrate for $45 \mathrm{~min}$ and stretched to a passive tension of $0.8 \mathrm{Gm}$. Investigation of the effects of $\mathrm{KCl}$ and norepinephrine on basal tone was performed without preconstriction of the rings. $\mathrm{KCl}$ was added in concentrations of 10,50 , and $118 \mathrm{mmol} / \mathrm{l}$. In some rings, constriction response to $10^{-7}-10^{-6} \mathrm{~mol} / \mathrm{l}$ norepinephrine was tested. Relaxation responses to $10^{-7}-10^{-3} \mathrm{~mol} / \mathrm{l}$ concentration of ATP, a NOS agonist, and to $S$-nitroso- $N$-acetyl penicillamine, a NO donor, were determined after preconstriction of the rings with $10^{-7}-10^{-6} \mathrm{~mol} / \mathrm{l}$ norepinephrine. Percentage relaxation was calculated by normalizing the ring tension observed with norepinephrine to $100 \%$, as described before (12). A total of 12 rings from 3 normal term ventilated lambs and 24 rings each from 6 PPHN lambs were used for the in vitro studies.

\section{Expression of eNOS and SOD Isoforms in the Lung}

A part of the right upper lobe was flash frozen in liquid nitrogen immediately after harvest of the lungs. Samples from unventilated normal lambs with or without exposure to betamethasone, ventilated normal term lambs, and ventilated PPHN lambs were included as controls. Samples were homogenized in modified radioimmunoprecipitation assay buffer $(11,12)$ and sonicated to break the cells, and insoluble debris was removed by centrifugation. Protein concentration was measured, and a $20 \mu \mathrm{g}$ aliquot of the protein was used for immunoblotting with antibodies to cytosolic copper zinc SOD, extracellular SOD, and MnSOD, and an aliquot of $40 \mu \mathrm{g}$ was used for immunoblotting with antibodies to eNOS. Each sample was also immunoblotted for -actin, used as the internal loading control. Autoradiograms were imaged with Adobe PhotoShop v5.5, and the relative band densities were quantified using NIH Image 1.62. Integrated optical densities for SOD isoforms, eNOS, and -actin were measured, and the ratios of $\mathrm{SOD} /$-actin and eNOS/-actin were calculated for each sample.

\section{DHE Fluorescence in PA Segments With Intact Endothelium}

Second-generation branches of PAs were dissected fresh from the lungs of saline- and betamethasone-treated PPHN lambs and an unventilated normal lamb. A 1-mm length of the artery was cut open, placed in 6-well plates with Hank's Balanced Salt Solution, and endothelium was exposed. The PA segments were incubated with Hank's Balanced Salt Solution alone or containing $100 \mathrm{U} / \mathrm{ml}$ of polyethylene glycol-SOD for $30 \mathrm{~min}$. Then, DHE $\left(10^{-5} \mathrm{~mol} / \mathrm{l}\right)$ and Hank's Balanced Salt Solution with or without $10^{-5} \mathrm{~mol} / \mathrm{l}$ ATP were added (12). In some studies, PAs from a normal unventilated fetal lamb were incubated for $45 \mathrm{~min}$ with $1 \mathrm{mmol} / \mathrm{l}$ xanthine plus $20 \mathrm{mU} / \mathrm{ml}$ xanthine oxidase (Sigma Chemical, St Louis, $\mathrm{MO}$ ) to generate $\mathrm{O}_{2}^{-}$as a positive control for DHE staining. After $15 \mathrm{~min}$, PA segments were washed, placed in microwells of 8 -well chamber glass slides, and coverslipped. DHE fluorescence of endothelium was visualized and imaged with a fluorescent microscope (Nikon Eclipse 600; Nikon, Melville, NY), equipped with a SPOT RT Slide camera and software (Diagnostic Instruments, Sterling Heights, $\mathrm{MI}$ ). Fluorescence was quantified using MetaVue software (Universal Imaging, Downingtown, PA).

\section{Immunofluorescence for 3-NT}

The right middle lobe of the lung was gently inflated with optimum cutting temperature compound (Ted Pella, Redding, CA) infused into the bronchus. The lobe was wrapped and placed on dry ice to firm up the optimum cutting temperature. Blocks were prepared by cutting 2-3-mm-thick sections and were frozen in liquid nitrogen. For 3-NT labeling, $10-\mu \mathrm{m}$ sections of the frozen tissue were cut and mounted on slides. Tissue was fixed in $100 \%$ acetone, washed, blocked with $5 \%$ bovine serum albumin, and probed with $5 \mu \mathrm{g} / \mathrm{ml}$ antinitrotyrosine antibody (Upstate-Millipore, Billerica, MA) overnight at $4^{\circ} \mathrm{C}$. In some studies, sections from normal unventilated lung were incubated with $5 \mu \mathrm{mol} / \mathrm{l}$ peroxynitrite for $5 \mathrm{~min}$ to increase 3-NT formation in the lung as a positive control for 3-NT. Sections were washed and incubated with $20 \mu \mathrm{g} / \mathrm{ml}$ goat antimouse immunoglobulin G (Alexa Fluor 488; Invitrogen-Life Technologies, Grand Island, NY), and fluorescence visualized using a Nikon Eclipse TE-300 fluorescent microscope with excitation at $485 \mathrm{~nm}$ and emission at $530 \mathrm{~nm}$. Fluorescent images of similar-sized PAs and airway were captured and quantified as described above.

\section{Statistical Analysis of Data}

All data are shown as mean $\pm S D$, except for ring tension studies, where data are shown as mean \pm SEM. We compared the changes in the hemodynamic and blood gas values over time between control and betamethasone-treated groups by two-way ANOVA and StudentNewman-Keuls post hoc test when a significant difference $(P<0.05)$ was noted by ANOVA. Densitometry ratios for western blots and DHE/ nitrotyrosine fluorescence in PAs between the groups were compared by ANOVA and Newman-Keuls post hoc test. Ring tension data were compared between different groups and at different doses by two-way ANOVA and Newman-Keuls post hoc test.

\section{ACKNOWLEDGMENTS}

We thank Satyan Lakshminrusimha at the University of Buffalo for his valuable suggestions on the ventilation study protocol.

\section{STATEMENT OF FINANCIAL SUPPORT}

This work was supported by grants 1RO3 HD059076 and RO1 HL057268 from the National Institutes of Health, Bethesda, MD; a research grant from Ikaria, Clinton, NJ; grant UL1RR031973 from the Clinical and Translational Science Institute and Advancing Healthier Wisconsin Foundation of the Medical College of Wisconsin, Milwaukee, Wl; the Children's Research Institute of Children's Hospital of Wisconsin, Milwaukee, Wl; and the Muma Endowed Chair in Neonatology at Children's Hospital of Wisconsin, Milwaukee, WI.

\section{REFERENCES}

1. Fox WW, Gewitz MH, Dinwiddie R, Drummond WH, Peckham GJ. Pulmonary hypertension in the perinatal aspiration syndromes. Pediatrics 1977;59:205-11.

2. Walsh-Sukys MC, Tyson JE, Wright LL, et al. Persistent pulmonary hypertension of the newborn in the era before nitric oxide: practice variation and outcomes. Pediatrics 2000;105(1 Pt 1):14-20.

3. Konduri GG, Vohr B, Robertson C, et al.; Neonatal Inhaled Nitric Oxide Study Group. Early inhaled nitric oxide therapy for term and near-term newborn infants with hypoxic respiratory failure: neurodevelopmental follow-up. J Pediatr 2007;150:235-40, 240.e1.

4. Wilson KL, Zelig CM, Harvey JP, Cunningham BS, Dolinsky BM, Napolitano PG. Persistent pulmonary hypertension of the newborn is associated with mode of delivery and not with maternal use of selective serotonin reuptake inhibitors. Am J Perinatol 2011;28:19-24.

5. Winovitch KC, Padilla L, Ghamsary M, Lagrew DC, Wing DA. Persistent pulmonary hypertension of the newborn following elective cesarean delivery at term. J Matern Fetal Neonatal Med 2011;24:1398-402.

6. Ramachandrappa A, Rosenberg ES, Wagoner S, Jain L. Morbidity and mortality in late preterm infants with severe hypoxic respiratory failure on extra-corporeal membrane oxygenation. J Pediatr 2011;159:192-8.e3.

7. Berti A, Janes A, Furlan R, Macagno F. High prevalence of minor neurologic deficits in a long-term neurodevelopmental follow-up of children with severe persistent pulmonary hypertension of the newborn: a cohort study. Ital J Pediatr 2010;36:45.

8. Lakshminrusimha S, Russell JA, Steinhorn RH, et al. Pulmonary hemodynamics in neonatal lambs resuscitated with $21 \%, 50 \%$, and $100 \%$ oxygen. Pediatr Res 2007;62:313-8.

9. Steinhorn RH, Russell JA, Morin FC 3rd. Disruption of cGMP production in pulmonary arteries isolated from fetal lambs with pulmonary hypertension. Am J Physiol 1995;268(4 Pt 2):H1483-9.

10. Brennan LA, Steinhorn RH, Wedgwood S, et al. Increased superoxide generation is associated with pulmonary hypertension in fetal lambs: a role for NADPH oxidase. Circ Res 2003;92:683-91.

11. Konduri GG, Ou J, Shi Y, Pritchard KA Jr. Decreased association of HSP90 impairs endothelial nitric oxide synthase in fetal lambs with persistent pulmonary hypertension. Am J Physiol Heart Circ Physiol 2003;285:H204-11.

12. Konduri GG, Bakhutashvili I, Eis A, Pritchard K Jr. Oxidant stress from uncoupled nitric oxide synthase impairs vasodilation in fetal lambs with persistent pulmonary hypertension. Am J Physiol Heart Circ Physiol 2007;292:H1812-20. 
13. Steinhorn RH, Albert G, Swartz DD, Russell JA, Levine CR, Davis JM. Recombinant human superoxide dismutase enhances the effect of inhaled nitric oxide in persistent pulmonary hypertension. Am J Respir Crit Care Med 2001;164:834-9.

14. Lakshminrusimha S, Russell JA, Wedgwood S, et al. Superoxide dismutase improves oxygenation and reduces oxidation in neonatal pulmonary hypertension. Am J Respir Crit Care Med 2006;174:1370-7.

15. Chandrasekar I, Eis A, Konduri GG. Betamethasone attenuates oxidant stress in endothelial cells from fetal lambs with persistent pulmonary hypertension. Pediatr Res 2008;63:67-72.

16. Gao Y, Tolsa JF, Shen H, Raj JU. A single dose of antenatal betamethasone enhances isoprenaline and prostaglandin E2-induced relaxation of preterm ovine pulmonary arteries. Biol Neonate 1998;73:182-9.

17. Sadowska AM, Klebe B, Germonpré P, De Backer WA. Glucocorticosteroids as antioxidants in treatment of asthma and COPD. New application for an old medication? Steroids 2007;72:1-6.

18. Frank L, Lewis PL, Sosenko IR. Dexamethasone stimulation of fetal rat lung antioxidant enzyme activity in parallel with surfactant stimulation. Pediatrics 1985;75:569-74.

19. Walther FJ, David-Cu R, Mehta EI, Polk DH, Jobe AH, Ikegami M. Higher lung antioxidant enzyme activity persists after single dose of corticosteroids in preterm lambs. Am J Physiol 1996;271(2 Pt 1):L187-91.

20. Walther FJ, Jobe AH, Ikegami M. Repetitive prenatal glucocorticoid therapy reduces oxidative stress in the lungs of preterm lambs. J Appl Physiol 1998;85:273-8.

21. DeLemos RA, Shermeta DW, Knelson JH, Kotas R, Avery ME. Acceleration of appearance of pulmonary surfactant in the fetal lamb by administration of corticosteroids. Am Rev Respir Dis 1970;102:459-61.
22. Houfflin-Debarge V, Deruelle P, Jaillard S, et al. Effects of antenatal glucocorticoids on circulatory adaptation at birth in the ovine fetus. Biol Neonate 2005;88:73-8.

23. Grover TR, Ackerman KG, Le Cras TD, Jobe AH, Abman SH. Repetitive prenatal glucocorticoids increase lung endothelial nitric oxide synthase expression in ovine fetuses delivered at term. Pediatr Res 2000;48:75-83.

24. Suzuki K, Hooper SB, Wallace MJ, Probyn ME, Harding R. Effects of antenatal corticosteroid treatment on pulmonary ventilation and circulation in neonatal lambs with hypoplastic lungs. Pediatr Pulmonol 2006;41:844-54.

25. Farrow KN, Wedgwood S, Lee KJ, et al. Mitochondrial oxidant stress increases PDE5 activity in persistent pulmonary hypertension of the newborn. Respir Physiol Neurobiol 2010;174:272-81.

26. Morin FC 3rd. Ligating the ductus arteriosus before birth causes persistent pulmonary hypertension in the newborn lamb. Pediatr Res 1989;25:24550.

27. Abman SH, Shanley PF, Accurso FJ. Failure of postnatal adaptation of the pulmonary circulation after chronic intrauterine pulmonary hypertension in fetal lambs. J Clin Invest 1989;83:1849-58.

28. Perez M, Lakshminrusimha S, Wedgwood S, et al. Hydrocortisone normalizes oxygenation and cGMP regulation in lambs with persistent pulmonary hypertension of the newborn. Am J Physiol Lung Cell Mol Physiol 2012;302:L595-603.

29. Grover TR, Parker TA, Balasubramaniam V, Markham NE, Abman SH. Pulmonary hypertension impairs alveolarization and reduces lung growth in the ovine fetus. Am J Physiol Lung Cell Mol Physiol 2005;288: L648-54.

30. Liggins GC. Premature delivery of foetal lambs infused with glucocorticoids. J Endocrinol 1969;45:515-23. 